\title{
ANÁLISE DE DESEMPENHO EM REDES IEEE 802.3 APLICADO PARA SISTEMA DE TEMPO REAL
}

\author{
R. A. M. Valentim ${ }^{1}$, B. G. Araújo ${ }^{1}$, J. M. T. Lacerda ${ }^{1}$ e R. M. Medeiros ${ }^{1,2}$ \\ ${ }^{1}$ Universidade Federal do Rio Grande do Norte - Depto de Eng. Biomédica / ${ }^{1,2}$ Instituto Federal do Rio Grande do Norte \\ Campus de Mossoró \\ ricardo.valentim@ufrnet.br - brunogomes@dca.ufrn.br - jonymac@dca.ufrn.br - ronaldo.maia@ifrn.edu.br
}

Artigo submetido em maio/2011 e aceito em junho/2011

\section{RESUMO}

A tecnologia Ethernet domina o mercado de rede local de computadores. No entanto, não foi estabelecida como uma tecnologia para automação industrial, onde o determinismo procura os requisitos com um desempenho de tempo real. Muitas soluções têm sido propostas para resolver o problema do determinismo não, que se baseiam principalmente no TDMA (acesso múltiplo por divisão de tempo), passagem de token e mestreescravo. É neste contexto que este trabalho é realizado, através de medidas de desempenho em redes de comunicação que utilizam o padrão IEEE
802.3, observando o comportamento destas redes, quando submetidos a diferentes cenários de sobrecarga. Para isso, as variações foram aprovadas em ambiente de teste, que será baseado em Shared Ethernet (Hub), Ethernet e Ethernet Switch com prioridade (IEEE 802.1Q). Desta forma, é possível indicar quais os dispositivos analisados pelos testes de desempenho demonstrado um comportamento mais adequado para suportar as aplicações com requisitos de tempo real.

PALAVRAS-CHAVE: Ethernet de Tempo Real, Redes Industriais, Comunicação de Tempo Real.

\section{PERFORMANCE ANALYSIS NETWORK SYSTEM FOR IEEE 802.3 APPLIED TO REAL TIME}

\section{ABSTRACT}

Ethernet technology dominates the local net market of computers. However, it was not established as a technology for industrial automation, where the requirements demand determinism with a performance of real time. Many solutions have been proposed to solve the problem of the no determinism, which are based mainly on the TDMA (time division multiple access), token pass and master-slave. It is in this context that this paper carried through measures of performance on communication nets that use the standard IEEE 802.3, observing the behavior of these nets when submitted to different scenes of overload. For this, variations were adopted in the test environment, which will be based on Shared Ethernet $(H u b)$, Switch Ethernet and Switch Ethernet with priority (IEEE 802.1Q). This way, being capable to indicate which devices analyzed among the performance tests demonstrated a more appropriate behavior to support the applications with real time requirements.

KEY-WORDS: dance, social training and family. 


\section{ANÁLISE DE DESEMPENHO EM REDES IEEE 802.3 APLICADO PARA SISTEMA DE TEMPO REAL}

\section{INTRODUÇÃO}

Com o avanço tecnológico na área de eletrônica digital e a crescente demanda por aplicações distribuídas, o interesse por dispositivos de rede baseados em processadores tem aumentado de forma significativa (NOF, 2009). As redes industriais já fazem uso de nós inteligentes, ou seja, dispositivos de comunicação baseados em microprocessador que efetivamente são utilizados no controle de processos nas indústrias de manufatura. Para Nof (2009), isso ocorre devido à tendência de descentralização da computação que converge para um ambiente distribuído. Portanto, as funcionalidades passam a estar presentes em vários elementos de processamento mais simples, ao contrário da computação centralizada que encapsulava funcionalidades em poucos processadores com maior poder de processamento.

Diferente das redes de escritório, as redes industriais possuem aspectos mais específicos que Ihes atribuem requisitos mais exigentes, tais como: determinismo no tempo de resposta; requisitos críticos de tempo real; confiabilidade dos equipamentos e resistência a ambientes hostis, onde são encontrados elevados níveis de temperatura e perturbações eletromagnéticas.

No ambiente industrial, as aplicações muitas vezes decorrem de efetivamente de sistemas embarcados, sistemas de controle para processamento digital de imagens, monitoramento, interface homem máquina, etc. A comunicação entre estes nós tem exigências específicas que são completamente diferentes e muitas vezes opostas a aquelas encontradas em ambientes de escritório (DECOTIGNIE, 2001). Essas exigências constituem aspectos que compõem os requisitos básicos para redes industriais, tais como (VALENTIM et al., 2009):

- Comportamento temporal: determinismo, prioridade e tempo real;

- Confiabilidade: consistência de dados e redundância de dispositivos a fim de manter alta disponibilidade;

- Requisitos do meio ambiente: equipamentos específicos resistentes a ambientes hostil;

- Tipo de mensagens e volume de informações: as mensagens podem ter níveis de prioridade diferente e o seu volume pode determinar o tempo de ocupação do meio;

- Conectividade/Interoperabilidade: integração entre dispositivos e ativos de rede;

- Método de acesso a dados: cliente-servidor, produtor-consumidor, publisher-subscriber, dentre outros.

O comportamento temporal das redes industriais é uma das características essenciais, isso devido ao controle de processos nos quais, prioridade, determinismo e tempo real são aspectos imperativos. A Figura 1 apresenta um sistema hipotético de controle de temperatura onde o comportamento temporal é um requisito funcional do sistema, visto que, uma medição fora do prazo temporal (deadline) pode ocasionar um acidente e gerar riscos a vida humana e ao meio ambiente. 


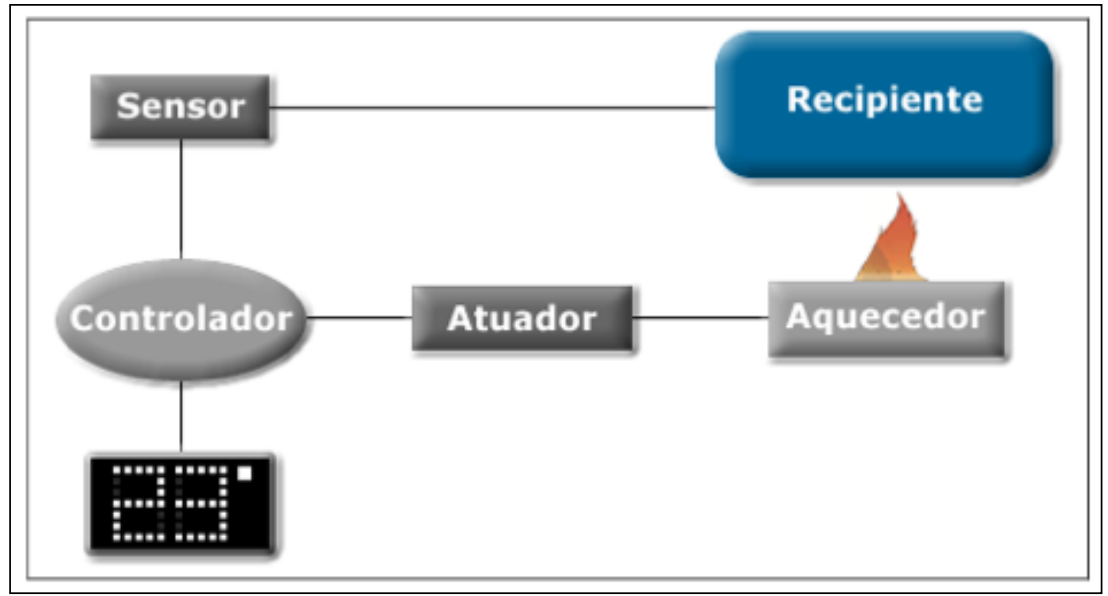

Figura 1 - Sistema de Controle de Temperatura em Tempo Real

Durante as últimas duas décadas foram desenvolvidas redes de comunicação para ambientes industriais que viabilizavam prover qualidade de serviço (QoS) a sistemas onde essa característica temporal é um fator relevante (PEDREIRAS et al., 2005). Genericamente, essas redes são chamadas de rede de barramento de campo, sendo frequentemente apropriadas à troca de pequenas quantidades de dados, sob as restrições de tempo, prioridade e confiabilidade (THOMESSE, 1999). Alguns exemplos bem conhecidos podem ser citados: Profibus, WorldFIP, Foundation Fieldbus, Controller Area Network (CAN) e DeviceNet.

Todavia, muitas dessas soluções são proprietárias, com pouca interoperabilidade e com taxas de transmissão algumas vezes incompatíveis com aplicações que necessitam de maior largura de banda. Esse fatores tornam tais tecnologias pouco atraentes. Como exemplo, é possível citar as aplicações voltadas a rede de campos na área de monitoramento de Poços de Petróleo, cujas informações atualmente estão demandando mecanismos de segurança mais eficazes, portanto, necessitando de um maior poder de processamento dos nós de rede, bem como, maior vazão de dados. Outro problema, pode ser verificado quando a política do processo de automação deve aplicar processamento de imagens. Neste caso as tecnologias de rede convencionalmente utilizadas no campo são insuficiente para atender esse tipo demanda. Um dos principais fatores para isso é sua baixa capacidade de prover a vazão de dados - isso quando comparada a tecnologias como a Ethernet, as quais possibilitam uma vazão de até $10 \mathrm{Gbps}$.

No sentido de solucionar esses problemas, existem esforços direcionados a pesquisas que estudam o uso de redes Ethernet como alternativa para área de automação industrial, os quais focalizam fortemente o comportamento temporal. No entanto, a tecnologia Ethernet não foi projetada para suportar os requisitos das redes de automação industrial, todavia, sendo uma tecnologia bastante interessante para este contexto, devido o seu alto desempenho, baixo custo, e sua expressiva interoperabilidade (DOLEJS et al., 2004), características que são requisitos fundamentais para atender as demandas atuais do processos de automação. 
Os aspectos que tornam a tecnologia Ethernet inadequada às aplicações industriais ficam em torno de dois pontos substanciais: ambiente hostil e o não determinismo gerado pela sua política de controle de acesso ao meio.

Com base em Brito et al. (2004), os problemas relacionados ao ambiente hostil são dirigidos às diferenças entre o contexto de um escritório e de uma indústria. $O$ ambiente industrial submete as redes a esforços mecânicos, de temperatura elevada e também de interferências eletromagnéticas. Porém, em relação ao fator ambiental as redes Ethernet já encontram soluções, pois já existem componentes Ethernet tais como: switches, hubs, cabos e conectores projetados para suportar tais características.

Segundo Carreiro et al. (2003), o problema da tecnologia Ethernet relacionado ao não determinismo é dirigido ao protocolo Carrier Sense Multiple Access with Collision Detection (CSMACD), dado que seu mecanismo de controle de acesso ao meio não é determinístico. Essa propriedade não determinística gerada pelo protocolo CSMA-CD (IEEE 802.3/ISO 8802-3, 1990), dificulta há predição do tempo para entrega dos pacotes, o que implica em atrasos aleatórios, característica não tolerada em sistemas de tempo real.

As colisões imprevisíveis geradas pelo CSMA-CD em segmentos Ethernet baseados em Hubs, impedem o determinismo temporal, onde o contexto das aplicações tem como aspecto restrições temporais críticas nas transmissões de dados. No entanto, os switches podem superar o problema de colisão, porém, existe o risco de contenção que pode ser gerado pelo grande número de pacotes enviados a uma mesma porta. Deste modo, conduzindo a atrasos ou descarte de quadros. De acordo com IEEE 802.1Q (IEEE-802.1Q, 1998), já existem switches baseados em QoS (Quality of Service) que estão melhorando em parte esta situação, porém ainda têm custos muito elevados (KISZKA et al., 2005).

Neste contexto, com o objetivo de resolver o problema do determinismo em redes Ethernet de modo a torná-la viável para as aplicações da automação industrial, existem pesquisas que trabalham basicamente sob duas diretrizes distintas: as que propõem modificações no controle de acesso ao meio físico (MAC) e outras que propõem implementações sob protocolos de alto nível, (BRITO et al., 2004), (PEDREIRAS et al., 2001) e (VIEGAS et al., 2006). Para tanto, podem ser referenciado como exemplos de pesquisas aplicadas ao controle de acesso ao meio para tornar determinística as redes Ethernet, o TDMA (Time Division Multiple Access) (BRITO et al., 2004), o TP (Token Pass), (VENKATRAMANI e CHIUEH, 1994), o VTPE (Virtual Token-Passing Ethernet) (CARREIRO et al., 2003) e o FTT-Ethernet (PEDREIRAS et al., 2005).

Dada a problemática exposta, e devido à relevância do tema para a área da automação industrial, este trabalho teve como motivação essencial realizar medidas de desempenho sobre o padrão IEEE 802.3. Deste modo, buscou identificar dentre os vários cenários analisados, qual apresentou o comportamento mais adequado ao ambiente dos sistemas industriais, que tem como premissa as restrições temporais. Para tanto, apresentando métricas extraídas a partir de experimentos, os quais permitiram analisar o comportamento temporal da rede quanto aos aspectos de desempenho e determinismo. Estes fatores são relevantes, pois apresentam parâmetros 
substanciais que indicam, portanto, qual a viabilidade e quais cenários o uso da tecnologia Ethernet na aplicação da automação dos processos industriais pode ser efetivamente aplicado de forma adequada.

Diante do objeto de pesquisa já supracitado, foram realizados estudos iniciais de medidas de desempenho em dois protocolos, UDP (User Datagram Protocol) (POSTEL, 1980) e RAW Ethernet (BARBATO e MONTES, 2004). Estas medidas previamente realizadas possibilitaram identificar qual o nível na pilha de comunicação (usando como referência o modelo OSI, (TANENBAUM, 2003)) mais adequado ao desenvolvimento de aplicações para redes de automação industrial, considerando o requisito de desempenho. Porém, tais, resultados não foram suficientes para subsidiar informações sobre o comportamento das redes Ethernet quando submetidas aos níveis de QoS exigidos na automação industrial.

Neste sentido, o presente artigo apresenta também medidas de desempenho sobre redes de comunicação que utilizam o padrão IEEE 802.3, observando o comportamento destas redes quando submetidas a diferentes cenários de sobrecarga. Para tanto, foi adotado variações no ambiente de teste, os quais foram baseados em Shared Ethernet (Hub), Switch Ethernet e Switch Ethernet com prioridade (IEEE 802.1Q), indicando, deste modo, qual dentre os ativos de rede analisados nos testes de desempenho demonstrou um comportamento mais apropriado para suportar as aplicações com requisitos de tempo real. Os experimentos realizados, bem como os resultados alcançados são relevantes para área da automação industrial, pois apresenta um conjunto de métricas extraídas a partir de uma tecnologia que poderá ser utilizada no desenvolvimento de aplicações para área da automação, as quais tenham restrições temporais nas faixas das aferições apontadas por meio dos experimentos realizados.

\section{MEDIDAS DE DESEMPENHO DOS PROTOCOLOS UDP E RAW ETHERNET SOBRE HUB E SWITCH}

Objetivo desta primeira análise foi subsidiar informação sobre o custo gerado na comunicação por meio do processamento das mensagens a nível da camada de transporte utilizando o protocolo UDP, quando comprado com processamento das mesmas mensagens no nível de enlace utilizando o protocolo RAW Ethernet

\section{Projeto Experimental}

Os experimentos realizados têm como objetivo medir o desempenho da tecnologia Ethernet utilizando dois protocolos (UDP e RAW Ethernet). Efetivamente, as medidas de desempenho foram conduzidas por uma metodologia aplicada na coleta e análise de dados, a qual será abordada ao longo dessa seção. A fim de viabilizar os experimentos propostos, foi utilizado duas estruturas para configurar o ambiente de teste: hardware e software. 
A estrutura de hardware é composta por dois microcomputadores similares interligados por uma rede Ethernet utilizando um ativo de Rede (Hub ou Switch), conforme ilustrado na Figura 2.

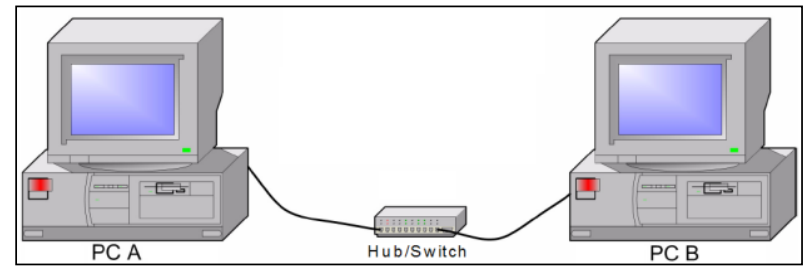

Figura 2 - Ativos de Rede Utilizados no Experimento.

As configurações utilizadas no ambiente ilustrado na Figura 2 foram:

- Processador: Intel Pentium III, 800Mhz;

- Memória RAM: 128 MB SDRAM DIMM;

- Placa mãe: Soyo modelo 7VBA133;

- Placa de rede: $10 / 100$ Mbps, Realtek;

- Hub: 10Mbps, 3Com, SuperStack II Hub 10;

- Hub: 100 Mbps, 3Com, SuperStack II Hub 100;

- Switch: 10/100/1000 3Com, SuperStack III;

- Cabos: UTP Categoria 5E.

Nos microcomputadores foram instalados softwares desenvolvidos especificamente para os testes propostos neste capítulo. No PC-A foram instalados softwares Clientes que são capazes de enviar pacotes UDP e RAW Ethernet. No PC-B foram instalados softwares servidores que são capazes de receber e retransmitir pacotes UDP e RAW Ethernet para a aplicação cliente de origem.

A estrutura de software foi totalmente desenvolvida no sistema operacional Linux com kernel 2.6.12, utilizando-se da linguagem de programação $C$. Na subseção seguinte é detalhada a arquitetura destas aplicações implementadas para o teste.

\section{Arquitetura da Aplicação}

A fim de prover a análise de desempenho da rede Ethernet foram desenvolvidas duas aplicações:

- uma baseadas em UDP e

- uma baseadas em RAW Ethernet. 
A Figura 3 e a Figura 4 apresentam as arquiteturas dos softwares, mostrando os mecanismos de funcionamento utilizados nos testes. Um dos aspectos das aplicações é que as duas abordagens operam em camadas diferentes na pilha de protocolo, porém os seus fluxos de atividades são iguais. $O$ fluxo de atividade das aplicações é orientado à medição do tempo do Round Trip Time (RTT). Para tanto, foi implementado um relógio de medição nas aplicações clientes com precisão de microssegundos.

O relógio é acionado no instante que a função de envio de dados é chamada, deste modo se obtém o instante $\mathrm{T} 1$, e finalizado quando o mesmo dado enviado à aplicação servidora é recebido de volta pela aplicação cliente e, portanto, obtém-se o instante T2. Assim, o tempo de RTT de um dado pacote é igual à diferença entre $\mathrm{T} 2$ e T1, ou seja, $\mathrm{RTT}=\mathrm{T} 2-\mathrm{T} 1$.

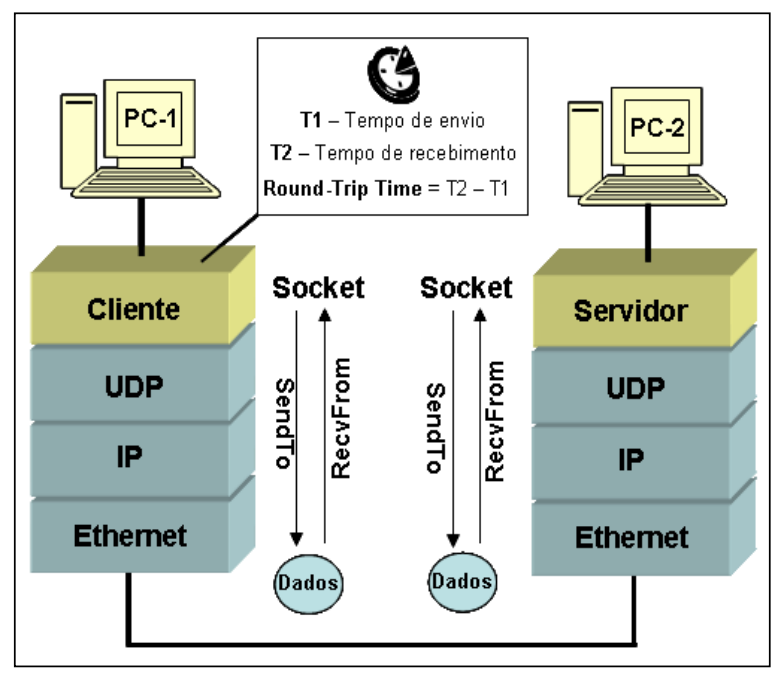

Figura 3. Arquitetura da Aplicação Baseada em UDP

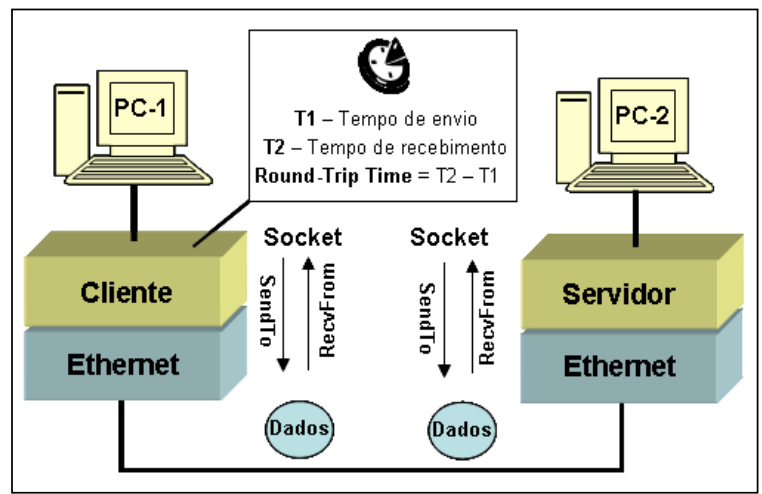

Figura. 4. Arquitetura da Aplicação Baseada em RAW ETHERNET.

Existem aplicações clientes que enviam dados para as aplicações servidoras. As aplicações servidoras recebem os dados e imediatamente devolvem os mesmos dados recebidos para as 
aplicações clientes. Esse procedimento foi adotado para garantir que os dados de ida e volta foram iguais, conforme ilustrado na Figura 3 e Figura 4.

Um fator substancial que diferencia estas aplicações em termos de arquitetura de software são os pontos de acesso na pilha de protocolo. No caso da aplicação da Figura 3, um socket é aberto sobre a camada de transporte (UDP). No caso da aplicação da Figura 4, um socket é aberto sobre a camada de enlace (RAW Ethernet). Esta estratégia foi adotada a fim de medir a diferença de desempenho de comunicação levando em consideração o overhead gerado para aplicações que trabalham no nível dois (enlace) e no nível quatro (transporte) do modelo de referência OSI.

A quantidade de vezes que os pacotes são enviados e seus respectivos tamanhos podem ser configurados na aplicação cliente, como, por exemplo: 1000 envios de pacotes de 512 Bytes. Na próxima subseção são descritos de forma detalhada os casos de teste que compõem a estratégia de medida de desempenho.

\section{Casos de Teste}

Os casos de teste utilizando o software desenvolvido foram aplicados em três cenários distintos:

- PC's interligados via Hub a $10 \mathrm{Mbps}$;

- PC's interligados via Hub a 100 Mbps;

- PC's interligados via Switch a 100 Mbps.

Em todos os cenários os pacotes de dados (payload) foram criados com os seguintes tamanhos em bytes: $64,128,256,512$ e 1024. Estes pacotes foram enviados do PC-1 para o PC-2 e retornados do PC2 para o PC-1. Cada experimento foi repetido 1000 vezes de modo a se obter resultados estatisticamente confiáveis.

Para cada experimento foram medidos os tempos médios de RTT utilizando o software de teste para os protocolos UDP e RAW Ethernet, onde se obteve a média aritmética e o desvio padrão. Com base em Kiszka et al. (2005), foram determinadas as medidas de desempenho apresentadas na Tabela 1 , onde $\mathrm{n}$ corresponde ao número de repetições do experimento. Verificou-se através dos experimentos que valores de $\mathrm{n}$ a partir de 500 são suficientes para se conseguir boa confiabilidade estatística, pois quando aumentado o tamanho de $n$, os tempos aferidos eram similares, o que foi verificado por meio do desvio padrão que não aumentava quando a quantidade mensagens era superior a 500, ou seja, não haviam alterações significativas no comportamento temporal dos experimentos. Para tanto, em todos os experimentos se utilizou $n=1000$, o dobro da amostra necessária. 
Tabela 1. RTT - Round Trip Time

\begin{tabular}{|c|l|}
\hline Variável & \multicolumn{1}{c|}{ Descrição } \\
\hline T1proc & $\begin{array}{l}\text { Tempo de processamento na pilha de } \\
\text { protocolo, somado ao tempo do NIC } \\
\text { (Network Interface Card) do PC-1. }\end{array}$ \\
\hline T2proc & $\begin{array}{l}\text { Tempo de processamento na pilha de } \\
\text { protocolo, somado ao tempo do NIC } \\
\text { (Network Interface Card) do PC-2. }\end{array}$ \\
\hline Tframe & $\begin{array}{l}\text { Tempo de propagação do quadro (Cabeçalho } \\
\text { e dados) no meio físico sobre Ethernet (ida e } \\
\text { volta). }\end{array}$ \\
\hline
\end{tabular}

Neste caso, é considerado que $\mathrm{T} 1$ proc $=\mathrm{T} 2$ proc, uma vez que as duas máquinas são similares. Os valores do tempo de processamento na pilha de protocolo foram medidos utilizando envio em loopback.

\section{Resultados Experimentais: UDP vs. RAW Ethernet}

Para os experimentos, procurou-se primeiramente verificar o tempo médio de processamento na pilha de protocolo. Para tanto, utilizou-se os envios de dados em loopback, como pode ser visualizado na Figura 5 e na Tabela 2, de onde se constatou que os tempos do RAW Ethernet para todos os tamanhos de pacotes enviados são praticamente constantes, em contraste com o comportamento observado no UDP que varia em função do tamanho do pacote de dados.

Na Figura 6 e na Tabela 2 são ilustrados o desempenho médio da rede Ethernet para os dois protocolos, utilizando ativos de redes com características diferentes (largura de banda e controle de acesso ao meio). Nesse experimento verifica-se que o desempenho do Hub de $100 \mathrm{Mbps}$ é superior ao Hub de 10 Mbps e também ao Switch de 100 Mbps.

Um fator importante notado neste experimento é que, para os dois ativos de rede com mesma taxa de transmissão (Hub e Switch a 100Mbps), os desempenhos para os protocolos foram bem diferenciados. No caso onde os pacotes eram de 1024 Bytes, o desempenho do Hub foi de aproximadamente $67 \%$ superior ao do Switch.

Para o caso onde os pacotes eram de 64 Bytes, o rendimento do Hub em relação ao Switch foi melhor em aproximadamente $25 \%$, independentemente do protocolo. Este fator é decorrente do 
tempo de processamento do Switch, que aumenta de acordo com o tamanho do pacote, o que não ocorre no Hub - e neste experimento os cenários de testes estavam livres de colisão.

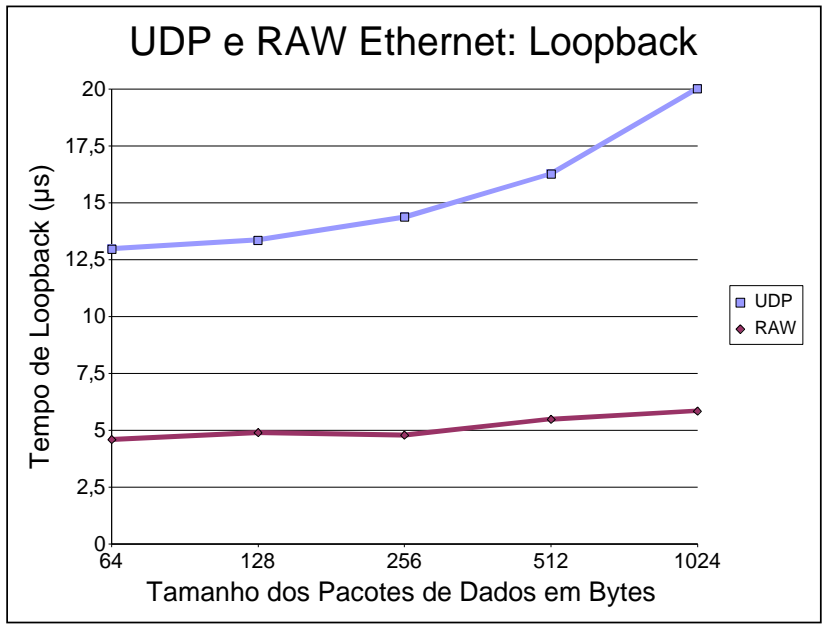

Figura 5. Loopback para UDP e RAW Ethernet.

Os resultados obtidos com estes experimentos mostram que o custo com overhead gerado pelo UDP (46 Bytes) é maior em relação ao RAW Ethernet (18 Bytes). Este custo se reflete na transmissão dos pacotes de dados e no tempo de processamento da pilha de protocolo. Deste modo, o UDP aumenta o tempo de processamento e também o custo de comunicação. Diante destes resultados, o melhor desempenho de comunicação verificado foi do RAW Ethernet utilizando Hub de 100Mbps.

\section{Análise dos Resultados}

Um dos principais pontos deste capítulo foi verificar os custos nos envios dos pacotes de dados, considerando o Hub de 100Mbps, uma vez que o mesmo demonstrou o melhor desempenho.

A metodologia utilizada foi segmentar o custo de comunicação em:

- SUT - Tempo de subida na pilha;

- SDT - Tempo de decida na pilha;

- NUT - Tempo de processamento do NIC (Subida);

- NDT - Tempo de processamento do NIC (Descida);

- HTX - Tempo de transmissão do cabeçalho (de acordo com protocolo utilizado, UDP ou RAW);

- DTX - Tempo de transmissão dos dados. 


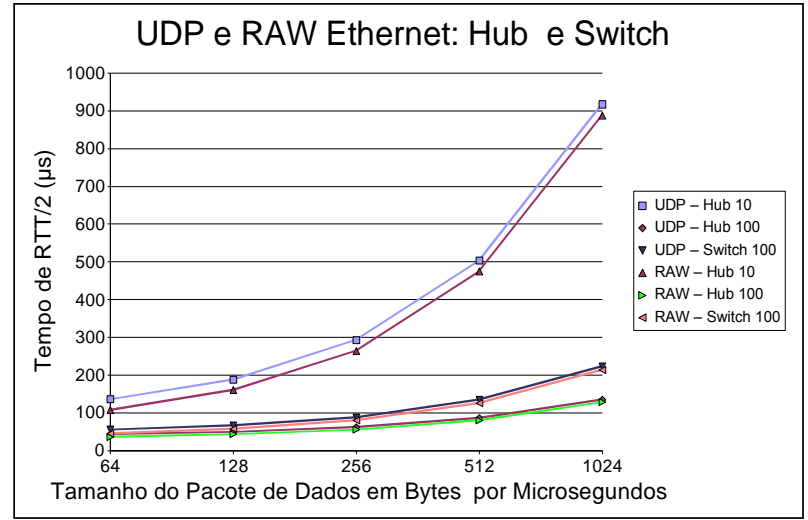

Figura 6. Comparação entre o UDP e RAW Ethernet.

Tabela 2. Medida de desempenho

\begin{tabular}{|c|c|c|}
\hline $10 \mathrm{mbps}$ (Hub) & UDP ( $\mu s)$ & RAW $(\mu \mathrm{s})$ \\
\hline 64 & 136 & 107,5 \\
\hline 128 & 188,5 & 160,5 \\
\hline 256 & 293,43 & 264,5 \\
\hline 512 & 503 & 474,5 \\
\hline 1024 & 917 & 887,5 \\
\hline $100 \mathrm{mbps}$ (Switch) & $\operatorname{UDP}(\mu \mathrm{s})$ & RAW $(\mu \mathrm{s})$ \\
\hline 64 & 55,54 & 46,07 \\
\hline 128 & 66,25 & 57,73 \\
\hline 256 & 88,55 & 79,7 \\
\hline 512 & 134,87 & 125,78 \\
\hline 1024 & 223,3 & 214,4 \\
\hline 100 mbps (Hub) & $\operatorname{UDP}(\mu \mathrm{s})$ & RAW ( $\mu \mathrm{s})$ \\
\hline 64 & 44,11 & 36,78 \\
\hline 128 & 49,4 & 43,31 \\
\hline 256 & 61,93 & 55,51 \\
\hline 512 & 86,87 & 80,22 \\
\hline 1024 & 134,99 & 128,37 \\
\hline Loopback & UDP ( $\mu \mathrm{s})$ & RAW ( $\mu s)$ \\
\hline 64 & 25,92 & 9,15 \\
\hline 128 & 26,7 & 9,77 \\
\hline 256 & 28,71 & 9,54 \\
\hline 512 & 32,51 & 10,94 \\
\hline 1024 & 40 & 11,67 \\
\hline
\end{tabular}

Os tempos de transmissão dos cabeçalhos (HTX) e dos dados (DTX) foram obtidos de forma teórica, considerando o envio em apenas um sentido, ou seja, do PC-1 para o PC-2. As outras variáveis foram obtidas através dos valores experimentais. Os tempos de decida (SDT) e subida (SUT) na pilha foram obtidos a partir dos experimentos de loopback. Os tempos de processamento dos NICs 
(Network Interface Card), os quais tem implicitamente incluído os preâmbulos ${ }^{1}$ dos quadros Ethernet, foram obtidos a partir da Equação $1^{2}$ e da Equação 2:

$$
\begin{aligned}
& \text { Frante ADQ }
\end{aligned}
$$

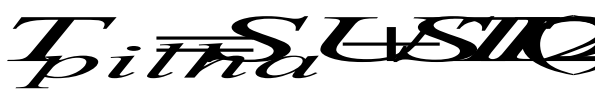

equação (1)

equação (2)

Logo, tem-se:

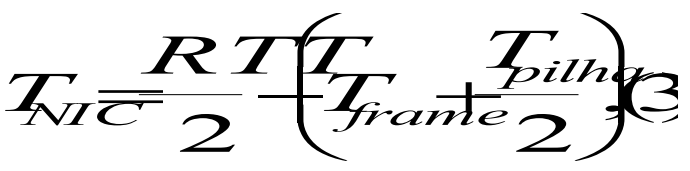

equação (3)

Assim:

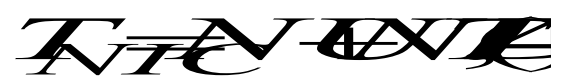

equação (4)

Na Figura 7 é ilustrado o custo com comunicação e processamento, onde se pode verificar que o custo do overhead do protocolo UDP é constante e superior em 55,5\% em relação RAW Ethernet. Também, foi observado que os custos de processamento na pilha e no dispositivo de rede estão relacionados respectivamente com a capacidade do processador e o desempenho do NIC.

Na Figura 8 é ilustrado o desempenho do Hub em relação ao Switch a 100Mbps. Como pode ser observado, o Switch aumenta o tempo de comunicação, isso é devido a seu custo de processamento, que aumenta proporcionalmente ao tamanho do pacote.

\footnotetext{
1 Formado de 8 Bytes, constituído de bits de " 0 " $s$ e " 1 "s alternados, serve essencialmente para sincronizar os receptores.

2 Para o Tframe foi considerado o tempo gasto apenas no envio do dado e não o RTT, pois para este experimento tinha-se necessidade apenas do custo de comunicação para enviar um dado de um destino a uma origem
} 


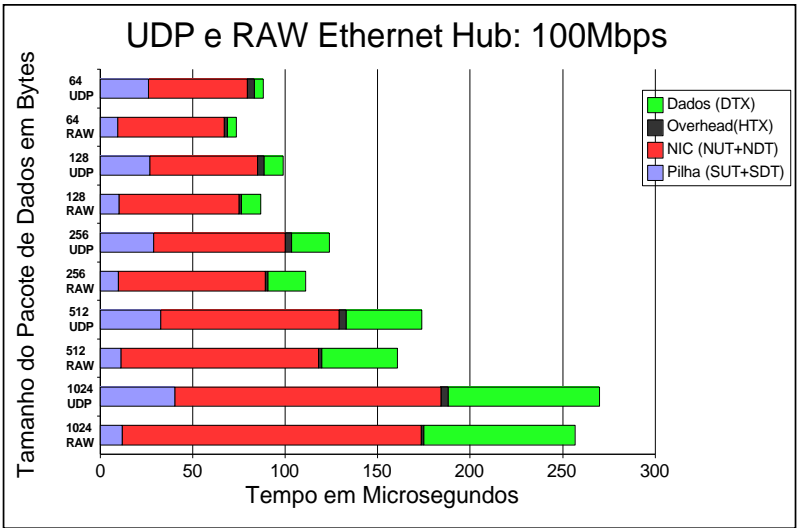

Figura 7. Custo do tempo de transmissão segmentado.

Nos experimentos realizados foi possível verificar que um dos maiores custos na comunicação ocorre nos NIC's (NUT e NDT). Outro fator observado é o menor overhead na pilha utilizando o protocolo RAW Ethernet, o que pode ser observado de forma mais evidente na Figura 7.

\section{Considerações: UDP vs. RAW Ethernet}

Os testes foram realizados em um ambiente onde os microcomputadores são dotados de um baixo poder de processamento, o que tipicamente ocorre em aplicações industriais embarcadas. Deste modo, validando a presente pesquisa para arquiteturas semelhantes.

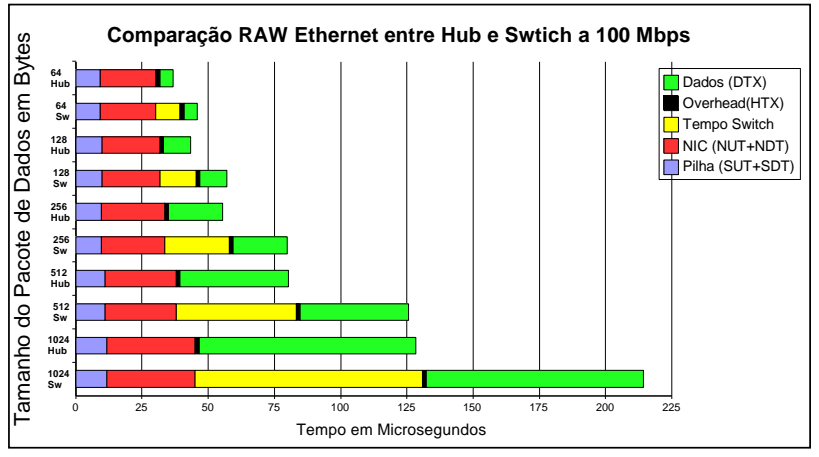

Figura 8. Tempos segmentados Hub Vs. Switch a 100Mbps.

Outra observação é que o Protocolo RAW Ethernet possibilita um ganho de desempenho de $55,5 \%$ em relação ao overhead gerado pelo protocolo UDP. Todavia, foi possível observar que o desenvolvimento de aplicações com RAW Ethernet aumenta em complexidade, pois sua API (Application Programming Interface) é de baixo nível, quando comparado à API socket UDP. 
Outro resultado significativo obtido com RAW Ethernet pode ser verificado nos testes com Hub a $100 \mathrm{Mbps}$ onde se obteve um desempenho entre $25 \%$ a $65 \%$ (variando de acordo com o tamanho do pacote) melhor que com Switch de $100 \mathrm{Mbps}$. Esta característica ocorre devido ao tempo de atraso imposto pelo mecanismo de enfileiramento do Switch aos pacotes.

Diante deste contexto, os experimentos realizados indicaram que RAW Ethernet é o protocolo mais adequado para o desenvolvimento de aplicações para redes Ethernet industrial do tipo IEEE 802.3. Isso porque o mesmo se mostrou mais eficiente em termos de tempo de comunicação (desempenho). Todavia, nestas medidas de desempenho, não foram realizadas aferições onde fosse possível avaliar o impacto de perturbações externas sobre as prioridades temporais dos fluxos de mensagens que utilizam à mesma tecnologia de comunicação, portanto, motivando novas medidas de desempenho de forma a contemplar aspectos de tempo real relacionado à tecnologia Ethernet de acesso ao meio.

\section{MEDIDAS DE DESEMPENHO SOBRE REDES IEEE 802.3}

Nesta seção é avaliado o desempenho de três tipos diferentes de tecnologias de comunicação (todas baseadas no padrão Ethernet), quando suportando um conjunto idêntico de fluxo de mensagens de tempo real num ambiente sujeito a perturbação externa. Esta análise de desempenho permitiu avaliar qual dentre as tecnologias de comunicação abordada apresenta um comportamento mais adequado ao desenvolvimento de sistemas com requisitos de tempo real. Para tanto, considerando um ambiente sujeito a perturbação de tráfego externo, o que não ocorreu nos experimentos realizados na seção anterior, posto que, nos experimentos realizados estavam livres perturbações de tráfego, pois o objetivo foi apenas o de analisar o desempenho entre os dois protocolos (UDP e RAW Ethernet).

\section{Escopo de Testes}

O escopo dos testes de medida de desempenho foi baseado no esquema ilustrado na Figura 9, apresentando três cenários de testes, os quais perfazem o ambiente onde foram realizadas as medidas de desempenho sobre cada um dos cenários descritos (Hub Ethernet a $10 \mathrm{Mbps}$, Switch Ethernet a 100 Mbps e Switch Ethernet com Prioridades (802.1Q com duas filas por porta) a $100 \mathrm{Mbps}$ ). Das sete estações ilustradas na Figura 9, foram utilizadas cinco estações para gerar tráfego (ET - Estação de trabalho) na rede, o qual foi baseada em uma distribuição uniforme de geração de tráfego. Esta metodologia é relevante para a análise de desempenho das tecnologias de comunicação utilizadas, isso porque, garante que não existirão instantes de baixa carga gerada sobre a rede. As outras duas são estações de tempo real (ETR), implementam um conjunto de fluxo de mensagens de tempo real que são trocadas entre ambas, sendo estas, as estações ETR1 e ETR2. 
O objetivo deste experimento foi verificar a perturbação causada pelo tráfego gerado pelas estações de trabalho ET1 a ET5 na comunicação entre as estações de tempo real. Deste modo, verificase o comportamento relativo de três diferentes tecnologias de comunicação baseadas no padrão Ethernet quando submetidas a variações de carga, e qual o impacto que estas variações de carga (de $10 \%$ a $60 \%$ da largura) terão sobre as propriedades temporais de um conjunto de mensagens de tempo real.

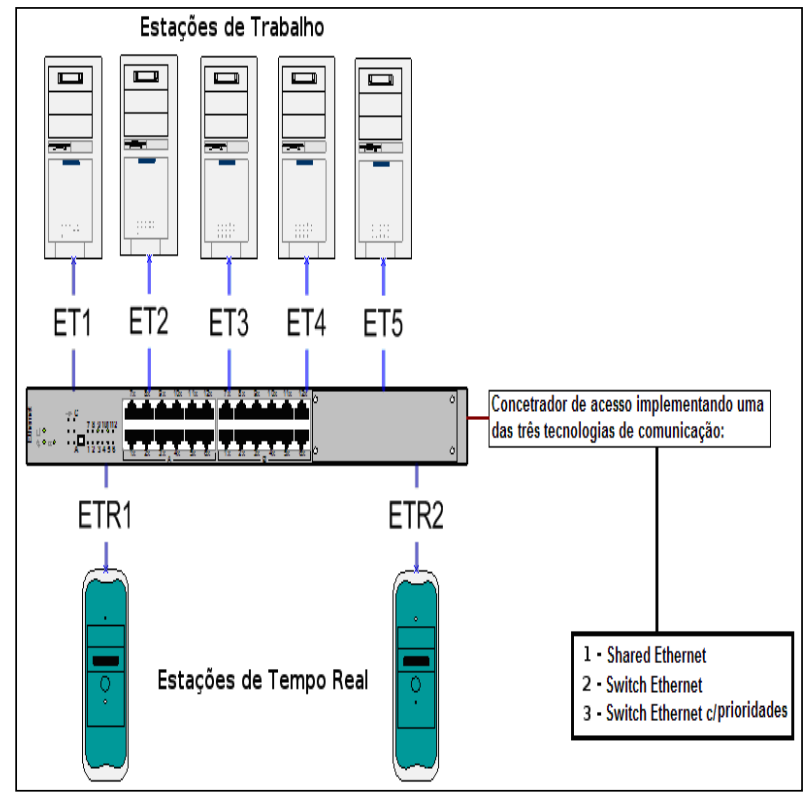

Figura 9. Sistemas de avaliação de desempenho.

\section{Configuração do Ambiente}

Para os testes de medida de desempenho foram utilizadas as seguintes configurações de hardware e software:

- Hardware:

- 7 (sete) estações do tipo PC;

- Processadores de $800 \mathrm{MHz}$ (ET) e $2.2 \mathrm{GHz}$ (ETR);

- Memória de $128 \mathrm{MB}(\mathrm{ET})$ e $1 \mathrm{~GB}$ (ETR);

- Placa de Rede 10/100 Mbps;

- Software:

- Sistema operacional em um versão genérica para as 5 (cinco) ET (Plataforma Windows);

- Sistema operacional Linux para as estações (ETR);

- Um gerador de tráfego utilizado nas estações (ET) desenvolvido sobre o protocolo UDP. 


\section{Cenários de Teste}

O cenário de testes de medição foi dividido em duas partes: A) geração de tráfego e B) testes de medição com ETRs, conforme pode ser observado na Figura 10. Na parte (A), estão as ETs responsáveis pela geração de tráfego na rede: ET1, ET2, ET3, ET4 e ET5, que irão gerar tráfego de acordo com perfis de carga necessário ( $10 \%$ a $60 \%$ da largura de banda). Para tanto, todo o tráfego gerado entre as respectivas ETs tem o mesmo destino (ETR1), de forma a obter a carga necessária à verificação do comportamento da rede de comunicação no que se refere ao tempo de resposta e ao Jitter (desvio padrão) das comunicações. Na parte (B), estão as ETRs que são responsáveis pelo suporte de fluxo de mensagens de tempo real, de onde efetivamente serão medidos os tempos. As ETRs irão realizar suas trocas de mensagens utilizando o mesmo mecanismo adotado na seção anterior para envio de dados utilizando o RAW Ethernet, ver Figura 4.

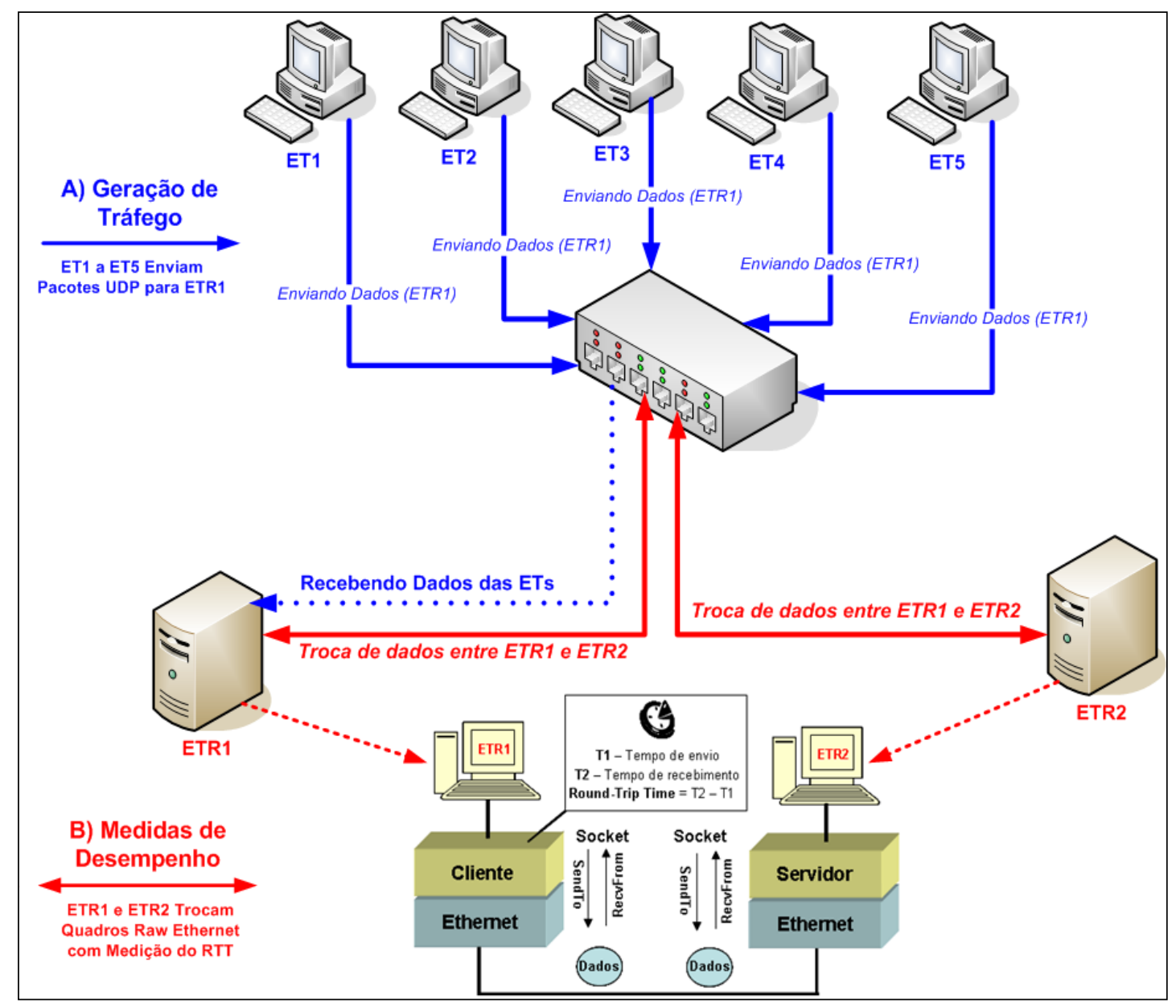

Figura 10. Envio de dados entre as estações 
Um aspecto importante deste cenário é que apesar das ETs abrirem Sockets UDP e enviarem mensagens a ETR1, a mesma não realiza a recepção destes, pois não foi criado receptores na ETR1 para os fluxos de dados enviados pelas ETs. Portanto, a carga é gerada na Interface de rede da ETR1, porém preservando o processamento, haja vista que os pacotes são descartados na própria interface de rede sem passar para o S.O (Sistema Operacional).

\section{Resultados Experimentais}

Nos experimentos realizados, além do Hub e do Switch convencional (Fast Ethernet - 100Mbps), foi utilizado um Switch com QoS para realizar as medidas de desempenho de tempo real. Este switch possui duas filas de prioridades.

No caso do Switch com QoS, foi necessário incluir no cabeçalho do quadro Ethernet os bits que indicavam a prioridade das mensagens enviadas. Estes bits foram inclusos através do Raw Ethernet, no instante da montagem das mensagens e apenas para às trocadas entre as ETRs, de modo que, estas tiveram maior prioridade sobre as mensagens enviadas pelas ETs.

A Figura 11 mostra os campos que foram utilizados para garantir o uso dos recursos de QoS providos pelo Switch utilizado neste experimento. Com base na norma IEEE-802.1Q (IEEE-802.1Q, 1998), atribuiu-se ao campo prioridade o valor 6 (seis), informado que o dado deve ser classificado com um dado de voz, no qual para o Switch utilizado representa um dado de maior prioridade. Embora o Switch disponibilize apenas duas filas de prioridade, o mesmo tinha uma abordagem de acordo com o padrão 802.1p para classificar os diferentes tipos de tráfego:

- $000=0$ : Best Effort;

- $001=1$ : Background;

- $010=2$ : Não Utilizado;

- $011=3$ : Excellent Effort;

- $100=4$ : Carga Controlada;

- 101 = 5: Vídeo;

- $110=6:$ Voz;

- 111= 7: Controle de Rede. 


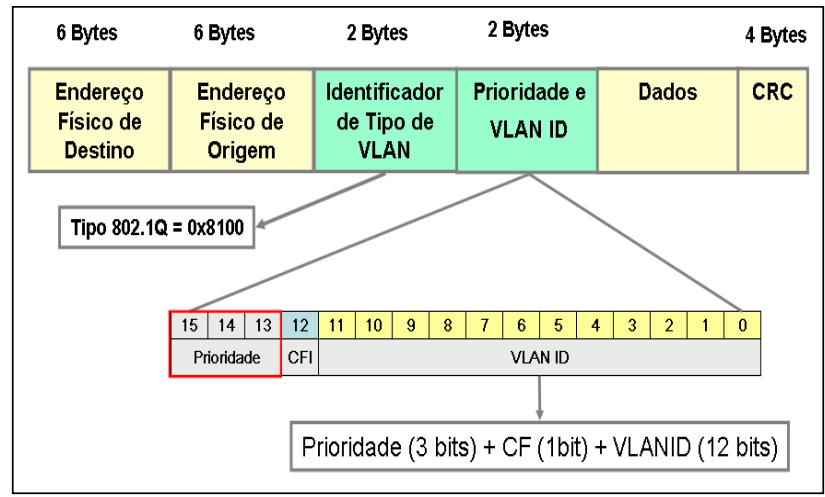

Figura 11. Formato do Quadro para 802.1Q, Fonte: (IEEE-802.1Q, 1998).

Todavia, estas classificações foram configuradas em dois perfis de tráfego, onde os valores entre 0 a 3 são classificados como baixa prioridade e os valores entre 4 e 7 são classificados como alta prioridade. Para tanto, estes valores deveriam está devidamente incluído no campo prioridade do cabeçalho Ethernet 802.1Q, conforme Figura 11.

\section{Descrição Geral dos Cenários Identificados}

Os cenários de testes foram dirigidos à avaliação do impacto de perturbações externas sobre as propriedades temporais dos fluxos de mensagens que compartilham a mesma tecnologia de comunicação. Portanto, avaliando qual das tecnologias de comunicação testadas é mais apropriada à comunicação de tempo real em ambientes de trabalho compartilhado (rede de não tempo real operando sobre o mesmo meio de acesso de uma rede de tempo real).

Neste ambiente os testes foram desenvolvidos diante da metodologia descrita a seguir.

○ Para as estações de trabalho na geração de tráfego (ET1....ET5):

- Rajada de dados: o número de pacotes por segundo a ser enviado pelas ETs. A rajada de dados tem relação com o tamanho do pacote (carga útil), largura de banda e carga a ser alcançada;

- Tamanho dos pacotes enviados pelas ETs foi de 1000 Bytes (carga útil), portanto variando apenas na rajada por segundo;

- Variação da carga: $10 \%, 20 \%, \ldots 60 \%$. A carga imposta a rede é igual ao somatório de toda a carga útil considerando os dados transmitidos pelas ETs, relativamente à taxa máxima de transmissão de dados suportada pela tecnologia Ethernet testada.

- Para as estações de tempo real:

- Tamanho dos pacotes $=64$ bytes (carga útil);

- Amostra 1000 pacotes (tamanho baseado nos experimentos anteriores citados nos seções anteriores, que demonstrou satisfatória qualidade estatística);

- Modo não promíscuo. 
Na Tabela 3 é apresentando de forma quantitativa o tráfego individual que cada ET gerou para garantir os cenários de carga desejados.

\section{RESULTADOS EXPERIMENTAIS}

Para melhorar a leitura dos resultados descritos, esta seção está dividida em subseções que abordam cada um dos cenários de teste propostos em relação à tecnologia Ethernet. Portanto, analisando duas medidas em relação à carga imposta na rede: Jitter (desvio padrão) e o Tempo de Resposta $\left(\mathrm{RTT}^{3}\right)$.

\section{Medidas Realizadas em Shared Ethernet (Hub)}

Os resultados em relação ao tempo de resposta mostram que na Ethernet compartilhada os valores de RTT aumentam de forma expressiva quando existe carga na rede, conforme pode ser observado na Figura 12. Um aspecto observado neste experimento é que, no caso onde a carga foi de $60 \%$, o tempo médio de resposta foi 9 vezes maior que no experimento realizado com ausência de carga.

As medidas de desempenho realizadas sobre a Ethernet compartilhada indicaram também que esta tecnologia tem um Jitter considerável, deste modo, elevando consideravelmente o seu fator de aleatoriedade. Este aspecto tem relação direta com as colisões geradas no meio físico, pois, quando ocorrem, acionam o algoritmo de back-off (Valentim, et. al, 2008), portanto, fazendo com que as estações escolham aleatoriamente slots de tempo para realizar o acesso ao meio.

Tabela 3. Tráfego gerado individualmente por cada ET para garantir a carga desejada

\begin{tabular}{|c|c|}
\hline \multicolumn{1}{|c|}{ Tecnologia } & $\begin{array}{c}\text { Carga para cada ET: } \\
\text { quantidade em bits } \\
\text { por segundo }\end{array}$ \\
\hline Hub Interligando as Estações (ETs e ETRs) a 10Mbps. & Para 10\%: $2 \times 10^{5} \mathrm{bps} ;$ \\
& Para $20 \%: 4 \times 10^{5} \mathrm{bps} ;$ \\
& Para $30 \%: 6 \times 10^{5} \mathrm{bps} ;$ \\
\hline
\end{tabular}

3 Tempo ida e volta: tempo total para que um pacote, datagrama, ou quadro deixe uma estação, atinja a outra e retorne a estação de origem. 


\begin{tabular}{|c|c|}
\hline & $\begin{array}{l}\text { Para } 30 \%: 8 \times 10^{5} \mathrm{bps} ; \\
\text { Para } 40 \%: 10 \times 10^{5} \mathrm{bps} ; \\
\text { Para 50\%: } 12 \times 10^{5} \mathrm{bps} ; \\
\text { Para } 60 \%: 14 \times 10^{5} \mathrm{bps} ;\end{array}$ \\
\hline $\begin{array}{l}\text { Switch Ethernet /Switch com Prioridade Interligando as Estações (ETs e ETRs) a } \\
\text { 100Mbps. }\end{array}$ & $\begin{array}{l}\text { Para 10\%: } 2 \times 10^{6} \mathrm{bps} ; \\
\text { Para 20\%: } 4 \times 10^{6} \mathrm{bps} ; \\
\text { Para 30\%: } 6 \times 10^{6} \mathrm{bps} ; \\
\text { Para 30\%: } 8 \times 10^{6} \mathrm{bps} ; \\
\text { Para 40\%: } 10 \times 10^{6} \mathrm{bps} ; \\
\text { Para 50\%: } 12 \times 10^{6} \mathrm{bps} ; \\
\text { Para 60\%: } 14 \times 10^{6} \mathrm{bps} ;\end{array}$ \\
\hline
\end{tabular}

Na Figura 13 é apresentado um gráfico que apresenta o Jitter (desvio padrão) entre os tempos de RTT em função do aumento de tráfego na rede. Um aspecto importante observado neste gráfico é que a inserção de carga na rede aumentou consideravelmente o Jitter quando comparado com os valores onde não houve geração de carga.

Outra característica observável na Figura 13 é um expressivo aumento no Jitter em função da carga, isso se justifica devido ao número de colisões, que probabilisticamente tem sua frequência aumentada quando cresce o número de pacotes na rede. Este aspecto apresenta-se como sendo o pior problema da Ethernet compartilhada para aplicações de tempo real, pois o tempo de resposta tornase mais imprevisível, o que é ruim para aplicações de tempo real, as quais têm como um dos principais requisitos a previsibilidade. 


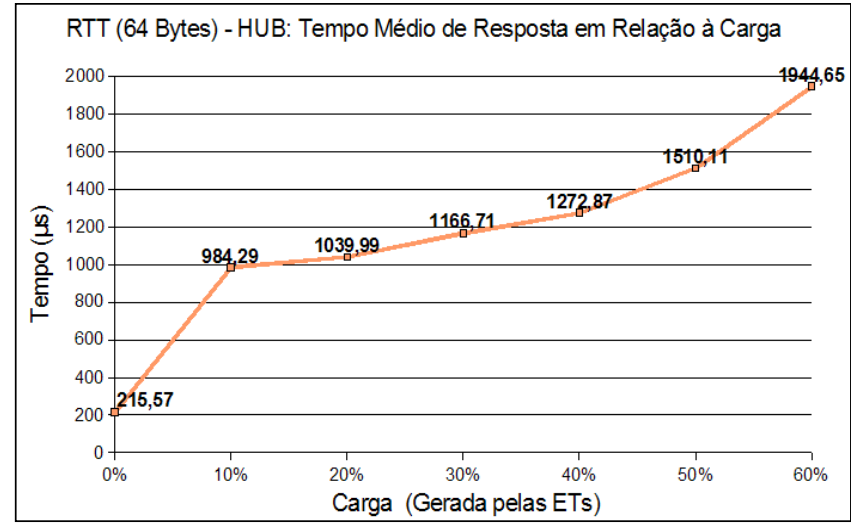

Figura 12. Tempo de resposta com Hub em função da carga.

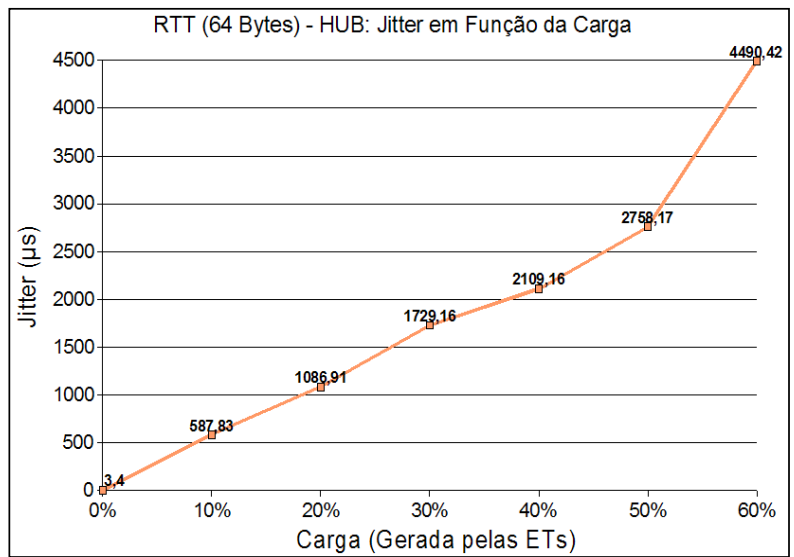

Figura 13. Jitter no Hub em função da carga.

\section{Medidas Realizadas em Switch Ethernet}

Os resultados obtidos com Switch em relação ao tempo de resposta são ilustrados na Figura 14. Um dos aspectos que podem ser observado neste gráfico é a variação entre o tempo de resposta com carga em $0 \%$ e $10 \%$. Neste caso, o tempo médio de resposta para uma carga de $10 \%$ é, aproximadamente, 2 vezes maior em relação à carga em $0 \%$. Este fator ocorre devido ao mecanismo de enfileiramento de mensagens dos Switches e também devido a atrasos gerados na placa de rede.

Os resultados obtidos com Switches para o tempo médio de resposta, se comparado com o Hub e levando-se em consideração os mesmo aspectos, há um ganho substancial de desempenho.

Neste mesmo contexto, observando os resultados obtidos com Switch e o comparando ao do Hub, é possível identificar um fator que torna mais notório o desempenho superior do Switch em cenários de carga. Para tanto, verificando os pontos de carga em $0 \%$ e $60 \%$, o tempo de resposta no Switch aumenta em 2,83 vezes, enquanto que, no Hub, o aumento é de 9 vezes. Deste modo, é 
possível verificar que os Switches sofrem um impacto menor quando submetidos a ambientes sujeitos as perturbações ocasionadas por tráfego da rede.

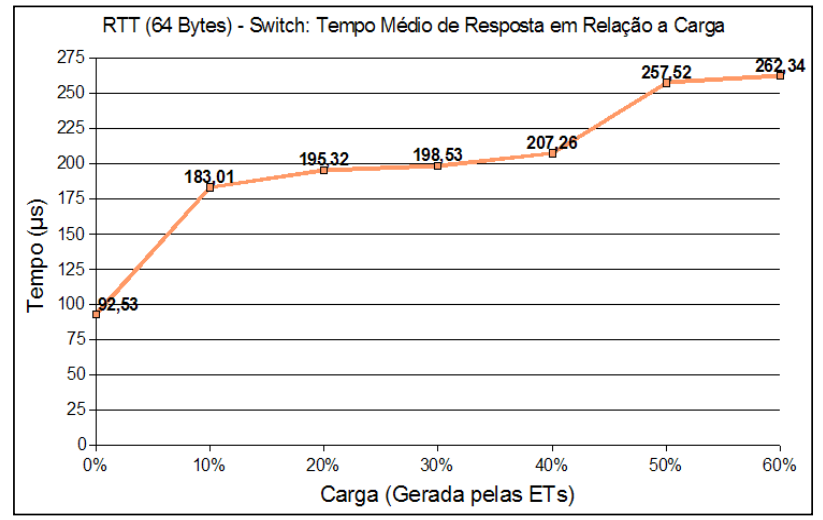

Figura 14. Tempo de resposta com Switch em função da carga

Na Figura 15 é ilustrado o Jitter das comunicações realizadas sobre o Switch. Um aspecto observado nas medidas realizadas com Switch foi uma redução considerável do Jitter quando comparado com o Jitter obtido com Hub.

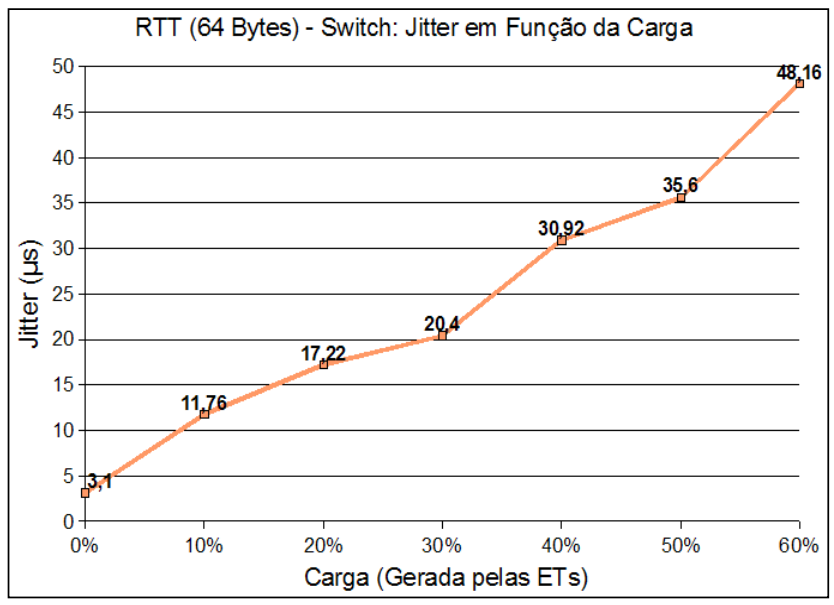

Figura 15. Jitter no Switch em função da carga

O gráfico ilustrado na Figura 15 também denota aspectos onde é possível verificar um aumento do Jitter em função da carga, por exemplo, quando a carga é de $10 \%$ o Jitter é aproximadamente 4 vezes maior se comparado com a carga em $0 \%$. Neste mesmo gráfico, também é verificado o aumento do valor do Jitter em 4 vezes para uma carga de $60 \%$ em relação ao experimento realizado com carga igual a $10 \%$. Este fator de aumento do Jitter em função da carga é um aspecto de 
não determinismo, haja vista que o aumento do Jitter reduz o grau de previsão quanto aos tempos de resposta.

Nos experimentos realizados, o Switch Ethernet demonstrou melhores resultados em relação ao Hub. Esta característica se dá devido a ausência de colisão, ou seja, os switches não fazem uso do protocolo CSMA-CD. Porém, por não apresentarem nenhum mecanismo de priorização de mensagens (QoS), o Jitter e o tempo de resposta das comunicações aumentam ou diminuem de forma não determinística, de acordo com a carga imposta na rede. Esta característica ocorre devido ao mecanismo de escalonamento dos switches (FIFO - First In First Out), o qual não contempla aspectos como fila de prioridades.

\section{Resultados obtidos com o Switch Ethernet 802.1Q}

O tempo de resposta do Switch com prioridade demonstrou melhor desempenho em relação ao Hub e ao Switch, o que é explicado pelo mecanismo de priorização de tráfego. Como observado na Figura 16 os tempos do Switch com prioridade aumenta em função da carga, porém todos os tempos são inferiores aos obtidos com os outros dispositivos avaliados.

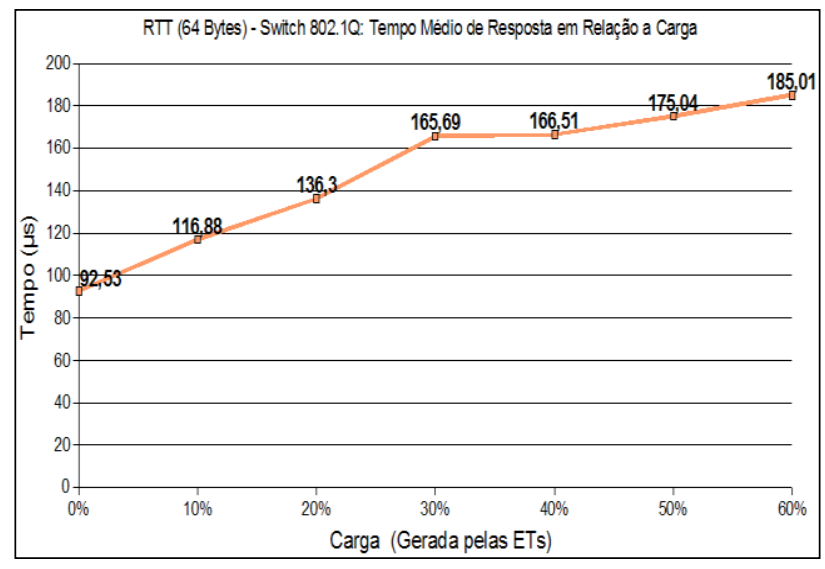

Figura 16. Tempo de resposta com Switch com prioridade em função da carga.

Um outro aspecto são os resultados referentes à variação do tempo de resposta, no qual demonstraram uma pequena variante no Jitter em função do aumento da carga, como pode ser verificado na Figura 17. Esta característica torna-se mais visível quando comparado aos resultados referentes ao Jitter obtido nos outros dispositivos analisados.

Comparando o Switch (a) e o Switch com prioridade (b) ambos entre $0 \%$ e $10 \%$ verifica-se que o aumento do Jitter em função da carga para (a) é de 3,80 vezes maior, enquanto que para (b) o aumento de 3,27 vezes maior, deste modo, mostrado um pequeno ganho. Porém, os ganhos mais expressivos se apresentam quando se observa o Jitter a partir de $10 \%$, onde em (a) a diferença do Jitter para as cargas de $10 \%$ e $60 \%$ é de $36,4 \mu \mathrm{s}$, enquanto para (b) é de $1,79 \mu \mathrm{s}$. Deste modo, mostrado 
que em situação de carga o Switch com prioridade mantém uma variação menor nos tempos de resposta. Portanto, probabilisticamente torna-se mais fácil de realizar predições, ou seja, este dispositivo é dotado de um maior fator de determinismo.

Assim, e com base nos valores analisados é possível verificar que os Switches com prioridade apresentam maior potencialidade a serem utilizados em ambientes onde existem demandas por aplicações de tempo real, haja vista, que apresentou um Jitter mais baixo em relação às outras tecnologias analisadas e também por demonstrar um bom desempenho diante dos cenários de carga apresentados. A fim de melhorar a leitura, nas Figuras 18 e 19 é ilustrada uma comparação entre Switch Ethernet e Switch com Prioridade, levando-se em consideração o tempo médio de resposta e o Jitter, respectivamente.

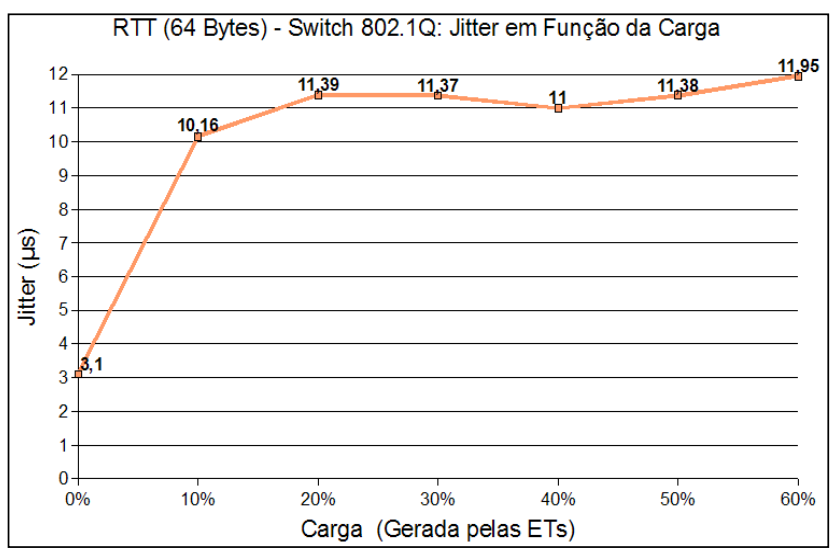

Figura 17. Jitter no Switch com prioridade em função da carga.

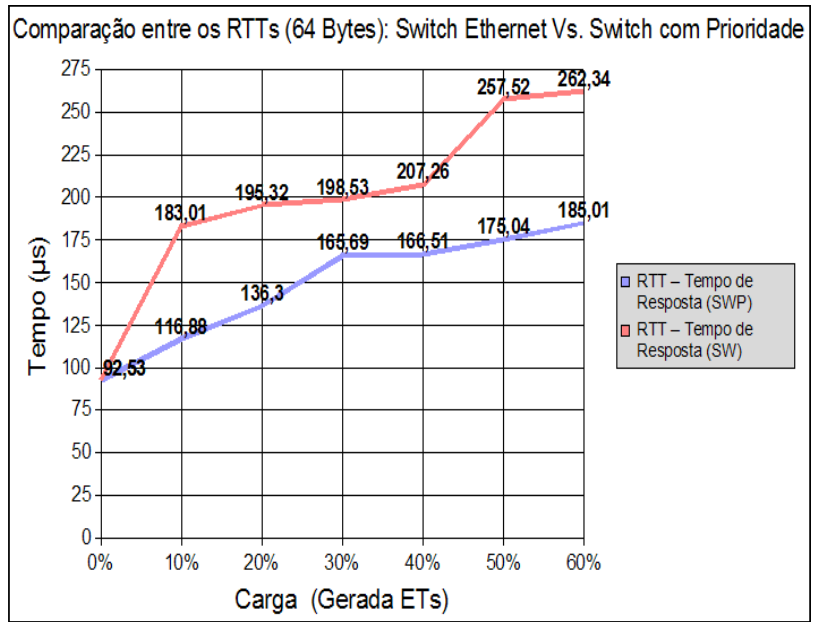

Figura 18. Comparação do RTT Médio entre Switch Ethernet (SW) e Switch com Prioridade (SWP). 


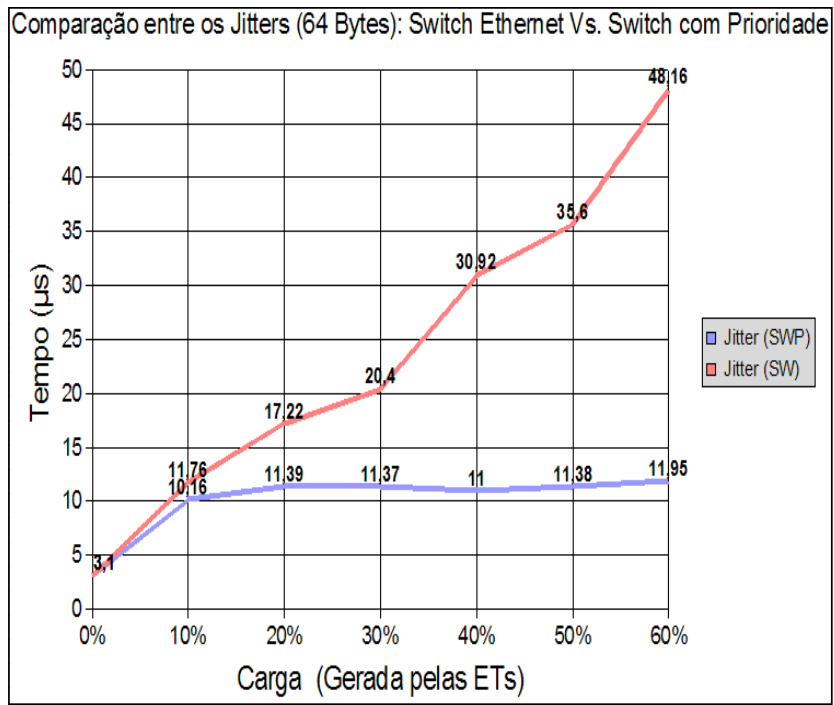

Figura 19. Comparação do Jitter entre Switch Ethernet (SW) e Switch com Prioridade (SWP).

\section{CONSIDERAÇÕES FINAIS}

Um aspecto substancial que pode ser observado neste artigo, e que pode ser verificado diante dos resultados apresentados é o potencial uso de aplicações de tempo real sobre Switches que implementam políticas de QoS em nível 2. Neste mesmo contexto os estudos realizados também apresentaram uma contribuição significativa, pois permitiu demonstrar que existem possibilidades factíveis de convivência entre tipos distintos de comunicação sobre um mesmo meio de acesso (utilizando Switch com prioridade), ou seja, comunicação de tempo real e comunicação sem requisitos de tempo real. Isso, sem alterar a política de controle de acesso das redes Ethernet.

\section{AGRADECIMENTOS}

Ao CNPq, CAPES, FINEp e ao Laboratório de Informática Industrial da Universidade Federal do Rio Grande do Norte (UFRN). 


\section{REFERÊNCIAS BIBLIOGRÁFICAS}

1. Nof, S. Y. 2009 Springer Handbook of Automation. 1st. Springer Publishing Company, Incorporated.

2. Barbato, L. G. C., Montes, A. "Técnicas de ocultação de Tráfego de Rede em Honeypots de Alta Interatividade" - ITA 60 Simpósio de Segurança em Informática - SSI'2004, Vol. 1, pp.1-10, São José dos Campos, SP, Brasil, 2004.

3. Brito, A. E. M., Brasileiro, F. V., Leite C. E., Buriti, A. C. Comunicação Ethernet em Tempo-Real para uma Rede de Microcontroladores, Anais do XV Congresso Brasileiro de Automática (CBA 2004) Brasil, setembro 2004.

4. Carreiro, F. Borges, Fonseca, J. Alberto, e Pedreiras, P, "Virtual Token-Passing Ethernet-VTPE", FET 2003 5th IFAC International Conference on Fieldbus Systems and their Applications, Aveiro, Portugal, July 2003.

5. Decotignie, J-D. "A Perspective on Ethernet as a Fieldbus". FeT'01, 4th Int. Conf. on Fieldbus Systems and their Applications. Nancy,France. Nov. 2001.

6. Dolejs, O., Smolik, P., e Hanzalek Z. "On the Ethernet use for real-time publish-subscribe based applications". In 5th IEEE International Workshop on Factory Communication Systems, Vienna, Austria, Sep. 2004.

7. IEEE 802.3/ISO 8802-3 - Information processing systems - Local area networks - Part 3: Carrier sense multiple access with collision detection (CSMA/CD) access method and physical layer specifications, 2nd edition, 21 September 1990.

8. IEEE-802.1Q - IEEE Standards for Local and Metropolitan Area Networks: Draft Standard for Virtual Bridged Local Area Networks, P802.1Q, January 1998.

9. Kiszka J., Wagner, B., Zhang, Y. and Broenink, J.F. "RTnet - A flexible Hard Real-Time Networking Framework", 10th IEEE International Conference on Emerging Technologies and Factory Automation, 19-22 Sept., Catania, 2005.

10. Pedreiras, P., Almeida, L. and Gai, P. "The FTT Ethernet protocol: Merging flexibility, timeliness and efficiency". Proceedings of the 14th Euromicro Conference on Real-Time Systems, Viena, Austria, June 19-21, 2001.

11. Pedreiras, P., Almeida, L., Gai, P. and Giorgio, B. "FTT-Ethernet: A Flexible Real-Time communication Protocol That Supports Dynamic QoS Management on Ethernet-Based Systems". IEEE Transactions on Industrial Informatics, Vol. 1, №. 3, August 2005.

12. RFC 768, Postel, J., "User Datagram Protocol", Network Information Center, SRI International, Menlo Park, Calif., August 1980.

13. Tanenbaum, A. S. "Computer Networks". 4rd. Ed., Prentice- Hall, 2003.

14. Thomesse, J.-P. "Fieldbus and interoperability" Contr. Eng. Pract., vol. 7, no. 1, pp. 81-94, 1999.

15. Valentim, R.A.M; Morais, A.; Guerreiro, A.; Branddo, G.B.; de Araujo, C.; , "MP-HA: Multicycles Protocol for Hospital Automation over multicast with IEEE 802.3," Industrial Informatics, 2008. INDIN 2008. 6th IEEE International Conference on, vol., no., pp.979-984, 13-16 July 2008. doi: 10.1109/INDIN.2008.4618244. 
16. Venkatramani, C. and Chiueh, T. "Supporting Real-Time Traffic on Ethernet". IEEE Real-Time Systems Symposium. San Juan, Puerto Rico. Dec 1994.

17. Viegas, R.; Valentim, R.A.M.; Texeira, D.G.; Guedes, L.A.; , "Performance Measurements of Protocols to Ethernet Real-Time Applications," Emerging Technologies and Factory Automation, 2006. ETFA '06. IEEE Conference on, vol., no., pp.1352-1355, 20-22 Sept. 2006. doi: 10.1109/ETFA.2006.384313. 